\title{
Implantación de un proyecto de mediación entre iguales: hacia una mejora de la convivencia en el contexto educativo
}

\author{
Implementation of a project of mediation among \\ equals: towards the improvement of coexistence \\ in the educational context
}

\author{
Elisa Isabel Galindo Alcalde, ${ }^{*}$ Aida Sanahuja Ribés ${ }^{* *}$
}

Recibido: 5 de abril de 2020 Aceptado: 18 de septiembre de 2020 Publicado: 31 de enero de 2021

To cite this article: Galindo, E. I. y Sanahuja, A. (2021). Implantación de un proyecto de mediación entre iguales: hacia una mejora de la convivencia en el contexto educativo. Márgenes, Revista de Educación de la Universidad de Málaga, 2 (1), 130-147 DOI: $\underline{\text { https://doi.org/10.24310/mgnmar.v2i1.8459 }}$

\section{RESUMEN}

El principal propósito de este trabajo se centra en la descripción de la implantación y evaluación de un proyecto de mediación entre iguales en un Instituto de Educación Secundaria, desde la posición del orientador educativo como coordinador del proyecto. Este trabajo se ha realizado durante el curso académico 2017/2018 en un IES de la provincia de Castellón. La implantación de la mediación en el centro se ha articulado dentro de un Seminario de formación del profesorado, a través del cual, se ha modulado un programa de formación para el alumnado en la mediación entre iguales, así como todas las decisiones referidas a la organización, coordinación y evaluación de la mediación en el centro. Los resultados reflejan la adquisición de habilidades y competencias por parte del alumnado que ha realizado el programa de mediación referido a la resolución de conflictos, el autocontrol y la técnica de la mediación propiamente dicha. Finalmente, resaltar la importancia de la figura del orientador como coordinador de todo el proceso. Este ha dispuesto de un espacio y tiempo privilegiado para las funciones de coordinación, convirtiéndose en una pieza clave para el buen desarrollo del proyecto.

Palabras clave: convivencia; educación secundaria; mediación entre iguales; orientación educativa

\section{ABSTRACT}

The main aim of this study is to describe the implementation and evaluation of a project of mediation among equals in a secondary school, from the Educational Counsellor's perspective as the Project Coordinator. This work has been developed during the academic year 2017/2018 in a secondary school in the Province of Castellón. The implementation of the mediation in the school has been articulated in a Teacher Training Seminar, through which a Student Training Program in Mediation among Equals has been expounded; as well as all the decisions related to the organization, coordination and evaluation of the mediation in the school. The results reflect the acquisition of abilities and skills by those students who have completed the program of me- 
diation, concerning conflict resolution, self-control, and the mediation technique itself. Finally, it is important to highlight the importance of the role of the Counsellor as the coordinator of the whole process. He has had a privileged space and time in order to perform the coordination tasks, becoming the cornerstone for the proper development of the Project.

Keywords: cohabitation; secondary education; peer mediation; education guidance

\section{INTRODUCCIÓN: LA CONVIVENCIA EN EL CONTEXTO EDUCATIVO}

Un aspecto relevante, en la Educación Secundaria Obligatoria, para la convivencia escolar es el modo de resolver los conflictos. Diversos trabajos plantean las bondades de la mediación como una herramienta para la resolución de conflictos (García-Raga y Grau, 2017; Silva y Torrego, 2016; García-Raga, Chiva, Moral y Ramos, 2016; Villanueva, Usó y Adrián, 2013). De tal modo, Villanueva et al. (2013) destacan que la mediación produce un efecto preventivo en la escalada negativa que se suele desarrollar en la violencia escolar, mejorando las actitudes pro-víctima y creando una cultura participativa y democrática.

En la actualidad la convivencia sigue siendo un reto para el sistema educativo (García-Raga et al. 2016). Aunque el origen de su estudio se remonta a los años 70, con el estudio de Olweus en Suecia (Olweus, 1998). Su mayor impulso aparece a partir de los 90 (Boqué, 2013; Torrego, 2017; Tuvilla, 2004, entre otros). Desde su origen hasta la actualidad, la convivencia ha pasado de ser un aspecto transversal, hasta convertirse en el centro de la educación (García-Raga y López, 2014). Un punto de inflexión en ese proceso de evolución fue el Informe Delors (Delors, 1996), donde uno de los principales pilares de la educación reside en aprender a vivir juntos, emplazando la convivencia en el centro de la atención educativa (García-Raga et al., 2016). Por tanto, el concepto de convivencia escolar va cobrando importancia hasta convertirse en el objetivo central del proceso educativo.

Para conseguir la mejora de la convivencia escolar, ciertas investigaciones (García-Raga et al., 2016) dan una gran importancia al impulso de estrategias eficaces que creen relaciones basadas en la deliberación, la interdependencia, el diálogo y la no violencia. Esto exige de profesionales que sean capaces de gestionar la convivencia en el contexto educativo (Observatorio Estatal de la Convivencia Escolar, 2009, Defensor del Pueblo, 2007). Entre dichas estrategias, la mediación es una de las que ha mostrado resultados más exitosos al convertir a los propios estudiantes en mediadores de los conflictos de sus compañeros (García-Raga y López, 2014).

Siguiendo a Torrego (2017), la mediación es un método de resolución de conflictos, en el que se recurre a una persona imparcial (mediador) para llegar a un acuerdo. El mediador orienta el proceso sin juzgar, creando un ambiente seguro que permita que los enfrentados lleguen a un acuerdo al que se sientan comprometidos (Alcover, 2006).

Por tanto, la mediación es una herramienta para la resolución de conflictos de forma pacífica que implica trabajar en un clima de entendimiento que permita la expresión de las emociones 
de forma libre y confidencial (Boqué, 2013). Por ello, la mediación es una técnica muy positiva para solucionar los problemas cuando las partes han de continuar su relación tras el conflicto (Torrego, 2017).

Siguiendo a Pulido, Martín-Seoane y Lucas-Molina (2013), la mediación entre iguales cobra sentido en sí misma, puesto que el alumnado se muestra más dispuesto a solicitar ayuda a sus iguales en lugar de solicitársela a un adulto.

Por su parte, desde el punto de vista del alumnado la mediación entre iguales tiene múltiples aspectos positivos. Cabe destacar que quien desempeña la función de mediador, ayuda a los demás y esto repercute positivamente en su enriquecimiento interior, debido al aprendizaje que realiza y al sentimiento de bienestar que les produce su labor como mediadores (Sánchez, 2013). Además, potencia sus competencias tanto personales como sociales (Silva y Torrego, 2016). El alumnado considera que la mediación es positiva en la prevención de la violencia y en la resolución de conflictos (García-Raga et al., 2016).

No obstante, según apuntan Ortega y Del Rey (1998), la mediación escolar tiene sus limitaciones, concretamente en los conflictos interpersonales en los que existe una asimetría de poder evidente, estos casos no deberían ser tratados mediante la mediación, ya que no está presente una condición imprescindible como es la relación equilibrada y la reciprocidad de las partes implicadas.

En la literatura y en la red hay publicados diversos programas que guían el proceso de implementación de la mediación en un centro educativo. A continuación, se realiza una sucinta descripción de los elementos más destacables de algunos de ellos.

Torrego (2017) en "Mediación de conflictos en instituciones educativas. Manual para la formación de mediadores”, presenta un programa completo con objetivos, contenidos, actividades y materiales distribuidos en seis módulos: 1) presentación e introducción al taller de mediación, 2) el conflicto y sus elementos, 3) la mediación, 4) habilidades para una comunicación eficaz, 5) experimentar la mediación y 6) la mediación en marcha. Propone una temporalización entre 16 y 20 horas según si se hacen todas las actividades propuestas o no.

Boqué (2013) en la "Guía de mediación escolar. Programa comprensivo de actividades de 6 a 16 años”, presenta un programa con una descripción, valoración y conclusiones de cada sesión, agrupadas en siete grandes bloques: 1) comprensión del conflicto, 2) comunicación abierta, 3) expresión de emociones y sentimientos, 4) habilidades de pensamiento, 5) participación activa, 6) convivencia pacífica y 7) el proceso de mediación. Desarrolla un total de 22 sesiones adaptadas a diversos grupos de edad: de 6 a 8 años, de 8 a 10 años, de 10 a 12 años, de 12 a 14 años y de 14 a 16 años.

La Junta de Andalucía en su guía "Mediación en la Resolución de conflictos y Programa de Alumnado Ayudante”, propone los pasos a seguir para la implantación de la mediación escolar en un centro educativo. Describe las fases de la mediación y el proceso en cada una de ellas.

El Gobierno Vasco en su guía “Cómo poner en marcha, paso a paso, un programa de mediación escolar entre compañeros/as” (Corbeña y Romera, s.f.), describe las fases para la implementación de un programa de mediación entre compañeros/as, estas son: 1) el compromiso del centro y la 
creación del equipo coordinador;2) el diseño del programa y del proceso de implementación; 3) la selección del equipo mediador; 4) la formación del equipo mediador; 5) la difusión del programa; 6) la organización del servicio; 7) la coordinación y el seguimiento y 8) finalmente la evaluación.

Siguiendo a García-Raga, Bo-Bonet, Boqué (2017), en la Comunidad Valenciana, el origen de la mediación es relativamente reciente. En el 2002 se inició la formación de diversos centros de Valencia y Castellón a través del “Centro de Formación, Innovación y Recursos Educativos” (CEFIRE). Concretamente, en los inicios se formaron 593 centros en Valencia y 450 en Castellón. De su estudio conviene destacar, que tanto la percepción del alumnado, como la mediación facilita el enfrentarse a la resolución de conflictos.

En cuanto a los resultados encontrados tras la aplicación de programas de mediación desde la perspectiva de la coordinación, investigadoras como García-Raga y Grau (2017) destacan por un lado la satisfacción de toda la comunidad educativa. Concretamente, recalcan los beneficios en el alumnado referidos al desarrollo personal, en la resolución de conflictos, el aumento de la autonomía, etc. En cambio, se encuentran dificultades en cuanto a la organización del tiempo y los espacios, así como mayor difusión del proyecto en sí mismo.

\section{OBJETIVOS}

El principal objetivo de este trabajo radica en describir una experiencia de implantación de un proyecto de mediación en un Instituto de Educación Secundaria (de ahora en adelante IES) con el propósito de mejorar la convivencia y las relaciones del alumnado del centro. Dicho objetivo general se concreta en los siguientes objetivos específicos:

Objetivo 1: Describir el proceso de implantación de la mediación entre iguales en un instituto de secundaria.

- Objetivo 1.1: Constituir en el IES un seminario con el profesorado encargado de realizar las actuaciones necesarias para la consecución de la implantación de un programa de mediación entre iguales.

- Objetivo 1.2: Articular y desarrollar el programa de mediación a través de la formación de alumnos mediadores.

Objetivo 2: Evaluar las acciones efectuadas durante la implantación de la mediación.

\section{CONTEXTO Y PARTICIPANTES}

El trabajo que aquí se presenta se ha llevado a cabo en un contexto real' ${ }^{1}$, es por lo cual no se revelará ninguna información.

1 En cumplimiento del Reglamento (UE) 2018/1725 del Parlamento Europeo y del Consejo, de 23 de octubre de 2018, relativo a la protección de las personas físicas en lo que respecta al tratamiento de datos personales por las instituciones, órganos y organismos de la Unión, y a la libre circulación de esos datos, y de la Ley Orgánica 3/2018, de 5 de diciembre, de Protección de Datos Personales y garantía de los derechos digitales. 
El IES está situado en la provincia de Castellón (España), imparte Educación Secundaria Obligatoria, Bachillerato, Formación Profesional de Grado Medio, Formación Profesional de Grado Superior y Formación Profesional Básica.

El centro cuenta con 650 alumnos, con un nivel sociocultural medio-bajo. El alumnado es multicultural, de origen nacional, marroquí, rumano, entre otras nacionalidades con menor incidencia (búlgaros, pakistaníes, libaneses y chinos). Los conflictos predominantes son relacionales, rumores, apodos, bromas pesadas, insultos, etc. Este tipo de conflictos si no son resueltos pueden derivar en un clima tóxico en el aula que impide el aprendizaje del alumnado, agresiones físicas, o incluso en el acoso escolar. La mayoría del alumnado resuelven los conflictos, dentro del contexto escolar, de forma violenta, ya sea a nivel verbal - física - relacional por lo que los conflictos no se resuelven de manera pacífica y tienden a prolongarse en el tiempo y entre el alumnado. La forma de atender hasta el momento estos conflictos ha sido a través del régimen disciplinario interior del centro por lo que el número de expedientes es muy elevado, con todo lo que ello comporta.

En cuanto a los participantes en el estudio conviene diferenciar los participantes en el seminario de formación del profesorado y los participantes en el propio programa de mediación entre iguales.

En el seminario han participado ocho profesoras y dos profesores del claustro, entre ellos la orientadora, la cual ejerció las funciones de coordinadora del seminario. El profesorado participante tiene una gran sensibilidad ante la problemática de la convivencia en el centro, pero reconoce que no tiene herramientas para resolverlos, por ello la motivación de la formación e imple mentación de la mediación entre iguales.

Seguidamente, en la tabla 1 se presentan los diferentes participantes en el seminario atendiendo a diferentes criterios, como son: sexo, departamento, antigüedad en el centro y la relación laboral.

Tabla 1. Participantes en el seminario

\begin{tabular}{cccc}
\hline SEXO & DEPARTAMENTO & $\begin{array}{c}\text { ANTIGÜEDAD EN EL } \\
\text { CENTRO }\end{array}$ & RELACIÓN LABORAL \\
\hline$V$ & valenciano & 15 años & funcionario de carrera \\
$M$ & valenciano & 5 años & funcionaria de carrera \\
$V$ & música & 2 años & funcionario de carrera \\
$M$ & inglés & 3 años & funcionaria de carrera \\
$M$ & inglés & 4 años & funcionaria de carrera \\
$M$ & informática & 14 años & funcionaria de carrera \\
$M$ & biología & 3 años & interina \\
$M$ & biología & 1 año & interina \\
$M$ & educación física & 2 años & funcionaria de carrera \\
$M$ & orientación & 1 año & interina \\
\hline
\end{tabular}

Por lo que refiere al programa de mediación entre iguales, el alumnado participante ha sido configurado, de manera voluntaria, entre el alumnado de $3^{\circ}$ de la ESO a $1^{\circ}$ de Bachillerato. El motivo de esta selección ha sido porque el grupo de profesorado concluyó que el alumnado de menor edad no estaba madurativamente preparado para ejercer de mediadores. El hecho de que sean voluntarios y no seleccionados por el profesorado tuvo algunas discrepancias entre los 
docentes, ya que algunos de los voluntarios habían tenido problemas de disciplina y conflictos entre los iguales. Desde el Departamento de Orientación se argumentó la importancia de que todo el alumnado que quisiera formara parte, ya que así todos se beneficiarían de aprender otras formas de resolver los conflictos sin el uso de la violencia.

A continuación, se presenta de manera desglosada el grupo de estudiantes que empezaron la mediación (ver tabla 2).

Tabla 2. Alumnado participante en el programa de mediación

\begin{tabular}{cccc}
\hline \multicolumn{4}{c}{ Alumnado que comienza el programa de mediación } \\
\hline Curso & Varón & Mujer & Total \\
$3^{\circ}$ ESO & 4 & 11 & 15 \\
$4^{\circ}$ ESO & 4 & 14 & 18 \\
$1^{\circ}$ BACA & 2 & 4 & 6 \\
$1^{\circ}$ BAHA & 1 & 5 & 6 \\
Total & 11 & 34 & 45 \\
\hline
\end{tabular}

\section{IMPLANTACIÓN DE UN PROYECTO DE MEDIACIÓN ENTRE IGUALES EN EL IES: HACIA UNA MEJORA DE LA CONVIVENCIA EN EL CONTEXTO EDUCATIVO}

\subsection{Seminario de formación del profesorado}

Al iniciar el curso 2017-2018 se propone a la orientadora del centro: coordinar la continuación del seminario y la implementación de la mediación entre iguales.

Para esbozar las acciones a realizar, se vuelve a proponer la realización de un seminario, titulado "Implantación de un programa mediación entre iguales", dentro del plan de formación del profesorado (PAF) coordinado por el Centro de Formación, Innovación y Recursos para el profesorado (CEFIRE).

El objetivo general del seminario se centra en mejorar la convivencia del centro educativo a través de la mediación entre iguales. Concretamente, los objetivos específicos del seminario son:

1. Articular cooperativamente un programa de formación en mediación entre iguales para el alumnado del centro.

2. Realizar sesiones de formación del profesorado, encargado de llevar a cabo las mediaciones iniciales y que acompañará al alumnado posteriormente.

3. Organizar la implementación de la mediación entre iguales.

4. Definir y mantener la estructura organizativa para dar una continuidad el próximo curso. 


\section{E X P E R I E N C I A S}

A continuación, en la tabla 3, se puede observar la secuenciación de las sesiones del seminario, así como sus objetivos y actividades abreviadas.

Tabla 3. Distribución de las sesiones del seminario de formación del profesorado

\begin{tabular}{|c|c|c|}
\hline \multicolumn{3}{|c|}{ SEMINARIO FORMACIÓN DEL PROFESORADO } \\
\hline SESIÓN & OBJETIVOS & ACTIVIDADES \\
\hline $1^{a}$ SESIÓN & $\begin{array}{l}\text { 1. Constituir el grupo de mediación. } \\
\text { 2. Revisar la formación del profesorado. } \\
\text { 3. Establecer la coordinación del seminario }\end{array}$ & $\begin{array}{l}\text { Discusión dialógica entre los miembros } \\
\text { del seminario. }\end{array}$ \\
\hline $2^{a}$ SESIÓN & $\begin{array}{l}\text { 4. Formar al profesorado en las cuestiones básicas de } \\
\text { la mediación. } \\
\text { 5. Repasar los documentos realizados en el seminario } \\
\text { anterior. }\end{array}$ & $\begin{array}{l}\text { Exposición teórica de las fases y habili- } \\
\text { dades necesarias para la mediación. }\end{array}$ \\
\hline $3^{a}$ SESIÓN & $\begin{array}{l}\text { 6. Afianzar los contenidos prácticos de la mediación: } \\
\text { elementos, fases, herramientas mediante simulacros. }\end{array}$ & Realización de simulacros de mediación. \\
\hline $4^{\text {a }}$ SESIÓN & $\begin{array}{l}\text { 7. Afianzar los contenidos prácticos de la mediación: } \\
\text { elementos, fases, herramientas mediante simulacros. }\end{array}$ & Realización de simulacros de mediación. \\
\hline $5^{a}$ SESIÓN & $\begin{array}{l}\text { 8. Planifıcar y organizar el Programa de Mediación } \\
\text { entre iguales. }\end{array}$ & $\begin{array}{l}\text { Explicación del Programa de mediación } \\
\text { de Torrego (2017). } \\
\text { Adaptación del Programa de Torrego } \\
\text { (2017) a las características del alumnado } \\
\text { y a la disponibilidad del profesorado. } \\
\text { Diseño de la difusión del programa de } \\
\text { mediación entre el alumnado. }\end{array}$ \\
\hline
\end{tabular}

\section{IMPLANTACIÓN DEL PROYECTO DE MEDIACIÓN ENTRE IGUALES}

9. Evaluar del programa de mediación entre iguales

6 SESIÓN 10. Programar la continuidad durante el próximo

Debate - discusión dialógica de los curso. temas propuestos.

11. Evaluar el seminario de formación del profesorado.

\subsection{Implantación del proyecto de mediación entre iguales}

Posteriormente, se pasa a presentar el programa de mediación entre iguales, diseñado y articulado en el seno del seminario anteriormente expuesto. Conviene señalar que el programa de mediación se basa en el manual "Mediación de conflictos en instituciones educativas" (Torrego, 2017) y se complementa con diferentes dinámicas y técnicas propias del aprendizaje cooperativo (Pujolàs, 2008). 


\section{E X P E R I E N C I A S}

Los dos principales objetivos del programa de mediación entre iguales son:

5. Enseñar las habilidades y estrategias que son necesarias para realizar la mediación entre iguales.

\section{Potenciar un clima de ayuda y cooperación entre el alumnado que realiza el programa.}

Seguidamente, en la Tabla 4, se presenta una síntesis con los objetivos y las actividades que se han trabajado en cada una de las 10 sesiones que han configurado el programa.

Tabla 4. Temporalización del programa de mediación entre iguales

\begin{tabular}{|c|c|c|}
\hline \multicolumn{3}{|c|}{ PROGRAMA DE MEDIACIÓN ENTRE IGUALES } \\
\hline SESIÓN & OBJETIVOS & ACTIVIDADES \\
\hline \multirow[t]{3}{*}{$1^{\text {a }}$ SESIÓN } & Presentar del programa de mediación entre & - Dinámica: “El ovillo de lana”(Pujolàs, 2008). \\
\hline & iguales. & - “Los dos Carteles” (20') (documento 1.2 Torrego 2017, p. 25). \\
\hline & Explorar las expectativas del alumnado. & - “El contrato" (25') (documento 1.2 Torrego 2017, p. 26). \\
\hline \multirow[t]{3}{*}{$2^{\mathrm{a}}$ SESIÓN } & Conocer el papel del conflicto en la vida, sus & - Dinámica: «GRUPOS» (Pujolàs, 2008). \\
\hline & distintos elementos y formas más habituales de & - Análisis de un conflicto. (1. Guía para analizar los conflictos Torrego, 2017, \\
\hline & enfrentamiento al mismo. & p. 32), (documento II. 1b Torrego, 2017, p. 33). \\
\hline \multirow[t]{4}{*}{ 3a SESIÓN } & Favorecer el desarrollo del estilo colaborativo & - Dinámica: “Un mundo de colores” (Pujolàs, 2008). \\
\hline & en la resolución de conflictos para la mejora del & - Explicación de los elementos de un conflicto, (documento II.2 Torrego \\
\hline & desarrollo personal y la inserción social. & 2017, p. 37). \\
\hline & & - Reflexión estilos de enfrentamientos (documento II.3 Torrego 2017, p. 43). \\
\hline \multirow[t]{4}{*}{$4^{\text {a }}$ SESIÓN } & Conocer la mediación desde una perspectiva & - Dinámica: “La rueda” (Pujolàs, 2008). \\
\hline & global y como una de las propuestas eficaces & - Actividad: "lluvia de ideas, ¿QUÉ ES MEDIACIÓN?" \\
\hline & para mejorar la convivencia en una institución & - “Ideas previas sobre la mediación” (Torrego 2017, p. 49). \\
\hline & educativa. & - “El caso de MANU y FERNANDO” (Torrego 2017, p. 56-60). \\
\hline \multirow[t]{3}{*}{ 5a SESIÓN } & Aprender las fases principales de un proceso & - Dinámica de grupo. \\
\hline & de mediación formal, su función y la manera de & - Actividad las fases de la mediación. \\
\hline & desarrollar cada una de ellas. & - Explicación de las fases de la mediación (Torrego 2017, p. 62-63). \\
\hline \multirow[t]{7}{*}{ 6 SESIÓN } & Reconocer nuestros estilos de comunicación y & - Dinámica: “La mirada” (Pujolàs 2008). \\
\hline & analizar cómo facilitan o dificultan los procesos & - Explicación de la importancia de la comunicación en la resolución de \\
\hline & de mediación. & conflictos (Torrego 2017, p. 69). \\
\hline & & - “Las doce típicas” (Torrego 2017, p. 74). \\
\hline & & - “Técnicas de la escucha activa” (Torrego 2017, p. 78). \\
\hline & & - "Te escucho" (Torrego 2017, p.79). \\
\hline & & - "Jabón neutro" (Torrego 2017, p. 83). \\
\hline \multirow[t]{4}{*}{$7^{\mathrm{a}} \mathrm{SESIÓN}$} & Conocer y entrenarnos en habilidades básicas & - Dinámica inicial. \\
\hline & de comunicación que nos ayuden a mediar en & - Explicación de los mensajes en primera persona (Torrego 2017, p. 85-86). \\
\hline & los conflictos. & - “Yoyó: situaciones” (Torrego 2017, p. 88). \\
\hline & & - “Compatibilidad de caracteres: situaciones” (Torrego 2017, p. 91-92). \\
\hline $8^{\text {a }}$ SESIÓN & Experimentar la mediación. & Simulación de varias mediaciones. \\
\hline \multirow[t]{3}{*}{ ga SESIÓN } & Conocer otras experiencias en mediación entre & Jornadas de mediación entre iguales Benicàssim. \\
\hline & iguales. & \\
\hline & Consolidar la formación recibida. & \\
\hline \multirow[t]{2}{*}{ 10 SESIÓN } & Experimentar la mediación. & - Simulación de varias mediaciones. \\
\hline & Evaluar el programa de mediación. & - Dinámica de evaluación: Felicite - propone - critique (Aguirre et al., 2018) \\
\hline
\end{tabular}




\section{INSTRUMENTOS Y PROCEDIMIENTO DE SISTEMATIZACIÓN DE LA EXPERIENCIA}

Seguidamente se va a realizar un sucinto repaso sobre los diferentes instrumentos o técnicas que se han utilizado para llevar a cabo el seguimiento y la evaluación de la implementación del programa de mediación en el IES.

\subsection{Observación participante}

En palabras de Ruiz Olabuénaga (2012), la observación en investigación científica consiste en contemplar de manera sistemática y minuciosa el determinado fenómeno educativo que se pretende abordar. Cabe señalar que la observación fue participante (Peña, 2011), dado que fue la propia coordinadora del proyecto (orientadora educativa) quien la realizó.

\subsection{Diario de campo}

Para efectuar el seguimiento de todo el proceso se ha realizado un diario de campo de las sesiones con el alumnado y de las sesiones del seminario del profesorado. Siguiendo a Albertín (2007) el diario de campo consiste en tomar nota de los diferentes sucesos o hechos que transcurren y que resultan especialmente significativos o reveladores. Así mismo, conviene apuntar que el registro de dichos sucesos o hecho permitió corregir y rectificar durante el transcurso algunas cuestiones metodológicas que no estaban funcionando correctamente (p.ej.: distribución de los tiempos). Estas modificaciones han sido coordinadas por el departamento de orientación.

\subsection{Felicito- Critico-Propongo}

La técnica Felicito-critico-propongo es una dinámica que surge de las escuelas Freinet, y sirve para recoger la satisfacción de los participantes con el fin de avanzar en el proceso realizado (Aguirre et al., 2018). Al terminar el programa de mediación con el alumnado se llevó a cabo dicha técnica para conocer aquellos aspectos a encomiar del programa, los que se debía mejorar y posibles propuestas.

\subsection{DAFO}

Según apuntan Aliaga, Gutiérrez-Braojos y Fernández-Cano (2018), la técnica DAFO permite analizar los factores internos y externos para construir de modo eficaz estrategias de mejora. El DAFO permite organizar de modo visual en una matriz $2 \times 2$ aquellos factores internos tanto positivos como negativos (Fortalezas-Debilidades), así como los factores externos (Oportunidades-Amenazas). Esta técnica se utilizó con el propósito de recoger las valoraciones del profesorado y la orientadora educativa sobre todo el proceso de implementación del proyecto de mediación en el IES.

\section{RESULTADOS}

Con el propósito de presentar los resultados alcanzados en este trabajo, de manera clara y ordenada, se abordarán a través de los objetivos que han guiado este estudio. 
A tenor del primer objetivo marcado conviene apuntar que ya se ha descrito de manera sintetizada cómo se ha implantado la mediación entre iguales en el IES (Objetivo 1). La estructura del seminario de trabajo, configurado por el profesorado, ha permitido articular cooperativamente un programa de formación en mediación entre iguales en el centro (Objetivo 1.1). Para ello, previamente, bajo la coordinación de la orientadora, el profesorado participante ha recibido una formación respecto a dicha temática. En cuanto al programa de mediación (Objetivo 1.2), conviene apuntar que el alumnado ha contribuido a enriquecerlo considerablemente, con sus comentarios y opiniones, esto ha hecho que en ocasiones se saliese del guion establecido o marcado para la sesión, dando respuesta así a las demandas e inquietudes del alumnado.

En general, la ejecución del programa ha transcurrido con algunas dificultades por falta de tiempo en algunas sesiones, no permitiendo la profundización necesaria para consolidar los aspectos tratados en la misma.

En la primera sesión, la mayoría del alumnado esperaba que el programa fuera provechoso, ya que tenían que hacer un esfuerzo extra fuera del horario lectivo, les preocupaba la idea de perder el tiempo. Por otra parte, muchos coincidieron en que querían que el grupo de mediación fuera un lugar donde se pudieran expresar libremente, dónde se respetaran los diversos puntos de vista y que fuera un grupo de apoyo.

En la segunda sesión, surgieron conflictos personales que el alumnado había tenido a lo largo de su escolaridad y que les había marcado. Destacaron los insultos-motes, repetitivos que llegan a mellar la autoestima y a entrar en episodios de ansiedad y tristeza y que sólo gracias al tiempo y a la ayuda de algún adulto habían conseguido superar. De tal forma, que se sentían mucho más fuertes y con más libertad para ser como son.

Esta sesión llevó a explorar qué pasaba en las aulas para que esto ocurriera, llegando a la conclusión de que imperaba la ley de la selva: “o comes o te comen”. Los que no insultaban en la primaria, a lo largo de primero empezaban a hacerlo "es lo que todos hacen" "si no lo haces eres el raro de la clase”, convirtiendo la agresión verbal en lo habitual. Ante estas respuestas, se intentó dar la vuelta al problema: de igual modo que los que no insultaban se habían contagiado de los que sí, ¿por qué no contagiar al revés?, dejando de insultar y tratando de resolver las diferencias hablando. Todos aceptaron la propuesta. En la tercera sesión, hubo dificultades para entender la práctica de los estilos de afrontamiento ya que les costaba ponerse en el papel adjudicado y pensar en resolver el conflicto de una determinada manera. En esta sesión se empezó a notar la escasez del tiempo para el desarrollo de las sesiones.

En la cuarta sesión, en la representación de la mediación informal salieron a relucir la forma habitual de resolver los conflictos de las alumnas que salieron como mediadoras y que no son precisamente un ejemplo que seguir. Una mediadora intentaba imponerse a través de la agresividad en un principio verbal, hasta llegar a la intimidación física. Esta representación tan inesperada, proporcionó muchos ejemplos para debatir sobre el modo en el que habitualmente muchos compañeros intentan resolver los conflictos, el papel del mediador como la antítesis a lo que se había representado: en tono, vocabulario, gestos, aproximación... 
La quinta sesión fue muy teórica sin apenas participación por parte del alumnado. En la sesión de evaluación final, resaltaron esta sesión como aburrida. En lo que refiere a la sexta sesión, se reflexionó sobre los conflictos que surgen en ocasiones por sólo mirar y la importancia de una buena comunicación para evitarlos.

En la séptima sesión, se reflexionó sobre la aplicabilidad de emitir los mensajes en primera persona ante algo que nos molesta. La mayor parte del alumnado estuvo de acuerdo con que era una forma de resolver los conflictos de forma pacífica, aunque no se veían haciéndolo, ya que cuando algo les molesta de verdad se enfadan tanto que no les saldría de manera natural. En ese momento se comenta la relación con las emociones y la importancia de reconocerlas y expresarlas de un modo controlado para poder gestionarlas correctamente. Además, se trató el tema emocional en el proceso de la mediación y su papel como mediadores en el control de la situación.

En cuanto a los simulacros de mediación trabajados en la octava y en la novena sesión, los estudiantes mediadores se apoyaban mucho en el guion de la mediación. No obstante, iban introduciendo algunas de las habilidades practicadas en las sesiones anteriores de forma espontánea, lo que indicó una asimilación de los contenidos expuestos.

En la décima y última sesión se asistió a las Jornadas CO-COMEDIANT de Benicàssim. Las alumnas que prepararon el CONTA'M (en castellano: CUÉNTAME) hicieron un breve resumen de toda la formación que habían recibido. Posteriormente, un alumno salió voluntario para responder al "pasa palabra”, donde explicó el motivo por el cual se había iniciado como mediador, puesto que había sido víctima de continuos insultos y humillaciones, y quería contribuir a evitar esas situaciones en los demás.

Una de las principales consecuencias del programa fue la formación de un grupo de alumnado mediadores. Conviene señalar que no terminaron el programa todos los estudiantes que lo empezaron. Empezaron un total de 45 alumnos y terminaron un total de 32 . Seguidamente, en la figura 1. se muestra una comparativa entre el número de alumnos que empezó el programa y el número de alumnos que lo terminaron.

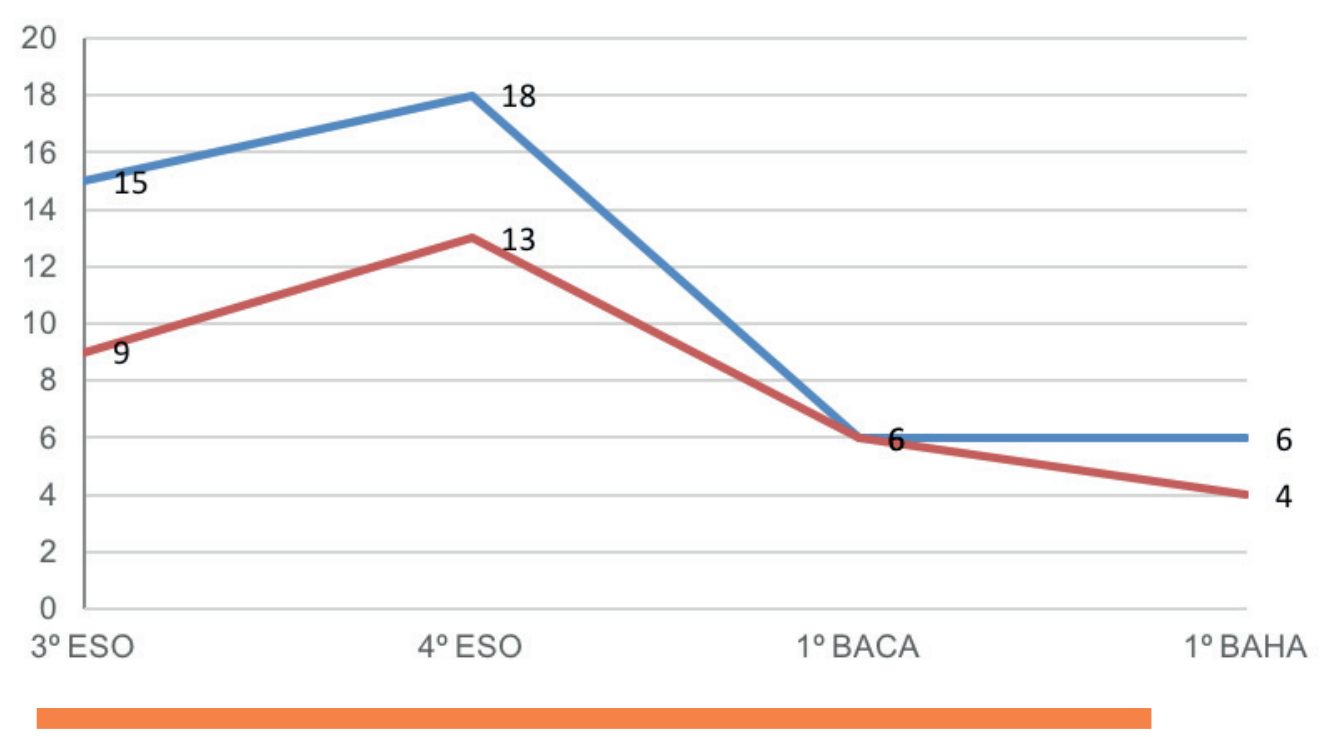

Figura 1. Comparativa entre el alumnado que inicia el programa y lo finaliza 
Como se aprecia en la Figura 1 (color azul para el no de alumnos que empezaron y rojo para el $n^{\circ}$ de alumnos que finalizaron), empezaron el programa 15 alumnos de $3^{\circ}$ de la ESO y lo terminaron 9. De $4^{\circ}$ de la ESO comenzaron 18 alumnos y lo terminaron 13 . En el caso de $1^{\circ}$ de bachillerato de ciencias (BAC grupo A) todos terminaron el programa y el $1^{\circ}$ de bachillerato de humanidades y ciencias sociales (BAH grupo A) empezaron 6 alumnos y terminaron 4. Los principales motivos de abandono del programa fueron: 1) falta de tiempo en el estudio de las materias académicas, 2) si faltaban más del $80 \%$ no se les daría título (una motivación era la obtención del certificado) y 3) por abandono del IES una vez cumplidos los 16 años.

Tal y como ya se ha explicado anteriormente para evaluar las acciones cometidas durante la implantación de la mediación (Objetivo 2) se efectuó la dinámica Felicito-Critico-Propongo realizada con el alumnado y el DAFO (uno realizado con todo el profesorado y otro hecho por la orientadora del centro).

Si nos centramos en las valoraciones que se desprenden de la dinámica Felicito-Critico-Propongo, vemos como el alumnado felicita en términos generales las dinámicas y la organización de las clases: "La forma de explicarlo”, “La organización y la dinámica”, "Dinámicas divertidas”. Así mismo, los estudiantes también han valorado el hecho de conocer mejor a los compañeros que han participado en el programa: "He conocido a más gente, una nueva experiencia muy divertida”, "En este curso, gracias a las actividades que se han hecho, nos hemos conocido mejor". También valoran positivamente los conocimientos que han adquirido con relación a la mediación y la convivencia en el contexto educativo: "Las clases han sido muy divertidas, ya que he aprendido cosas nuevas", "Hemos aprendido como controlar una situación delicada entre dos personas”, “Autocontrol”, “He aprendido a mediar”, "Nos han enseñado a solucionar problemas”. Con estos comentarios podemos corroborar que los objetivos específicos del programa, referentes a 1) enseñar las habilidades y estrategias que son necesarias para realizar la mediación entre iguales y 2) potenciar un clima de ayuda y cooperación entre el alumnado que realiza el programa, han sido adquiridos.

Las críticas del alumnado en cuanto al programa llevado a cabo giran en torno a las clases teóricas: "Había mucha teoría. Pero era parte del aprendizaje”, "Había demasiada teoría”, "Había clases un poco más aburridas". Otra crítica apunta a cuestiones del horario o del tiempo disponible en las sesiones: "El horario en el que se hace la mediación”, "El horario", "Ha sido demasiado corto”, “Poca duración”. Por último, señalar una de las críticas en referencia a mejorar y revisar los ejemplos de simulacros: "Los ejemplos de conflictos, a veces no son muy comprensivos o no hace falta acudir a la mediación”.

Finalmente, en cuanto a las propuestas el alumnado propone hacer más excursiones y salidas: "Más actividades fuera del centro", "Hacer más excursiones”, "Hacer más excursiones y salidas”, "Más comidas en grupo y excursiones”. Otro aspecto que proponen revisar atañe a la ampliación de sesiones, tanto en tiempo como en número: "Que haya más horas”, "Más clases”, "Podríamos hacer más clases y actividades diferentes”. Un estudiante propone la posibilidad de juntarse con los diferentes grupos de mediación para conocer mejora a todos los compañeros: "Propongo que se junten los grupos de mediación para que podamos conocer a más gente y sea más divertido”. Igualmente, los jóvenes proponen incrementar el simulacro de casos prácticos: "Realizar unas 
horas de mediación práctica”, "Hacer más prácticas en casos donde podamos mediar, ya que en la práctica hemos aprendido más”, "Hacer más prácticas”, “También nos gustaría hacer más simulacros para poder mejorar”.

Con la práctica parece que el alumnado adquiere mejor las competencias que en lugar de dar clases teóricas, que se les hacen más pesadas y densas: "Que las clases de teoría no se hagan tan largas”. Reclaman hacer una mejor difusión para que se difunda entre todos los compañeros: “Que expandan más la mediación entre los jóvenes”. También consideran relevante comenzar antes el programa para que existan repercusiones en el mismo curso académico en que se realiza: "Propondría darlo más a conocer y empezar antes el curso para aplicarlo en este".

En relación al DAFO efectuado por el profesorado y la orientadora cabe señalar como debilidades: el hecho de dedicar tiempo extra y la inexperiencia por parte del profesorado. Como amenazas: el cansancio por el sobreesfuerzo, la continua formación del alumnado mediador año tras año, dar a conocer los avances y que no parezca que no sirve de nada. Como fortalezas: destacar la mejora de la convivencia, la participación en el proyecto de los alumnos, la mejora de su autoestima y que vean las posibilidades que hay de cambiar las cosas. Y como oportunidades: subrayar la mejora de la gestión de conflictos, de la convivencia y del autocontrol gracias a la mediación del alumnado.

Paralelamente a la formación del alumnado, el profesorado ha realizado siete mediaciones estructuradas. Las temáticas de estas, así como la frecuencia del uso del servicio de mediación, se pueden observar en la tabla 5.

Tabla 5. Casos de mediación

\begin{tabular}{|c|c|c|c|}
\hline Caso & Parte A & Parte B & Acuerdo \\
\hline Caso 1 & $\begin{array}{l}\text { Se queja de que } B \text { le molesta. } \\
\text { Tras no aguantar más insulta a B }\end{array}$ & $\begin{array}{l}\text { No le daba importancia a su } \\
\text { conducta. No sabía que le } \\
\text { molestara. Antes los insultos de } \\
\text { A, empuja a B }\end{array}$ & $\begin{array}{l}\text { Ambos se comprometen a } \\
\text { pensar antes de actuar y ante } \\
\text { un conflicto hablar con el } \\
\text { tutor. }\end{array}$ \\
\hline Caso 2 & $\begin{array}{l}\text { Cree que B le ha insultado, } \\
\text { llevan juntas varios cursos y en } \\
\text { muchas ocasiones la insulta. Tras } \\
\text { pensar que le había insultado, A } \\
\text { insulta a B. }\end{array}$ & $\begin{array}{l}\text { A ha comentado algo de B pero } \\
\text { no es un insulto. Tras el insulto } \\
\text { de A la insulta. }\end{array}$ & $\begin{array}{l}\text { Se respetarán, no se } \\
\text { insultarán y no se molestarán. }\end{array}$ \\
\hline Caso 3 & $\begin{array}{l}\text { A tiene la percepción de que } B \text { se } \\
\text { ha reído de ella y A se ha } \\
\text { ofendido mucho y le hizo un } \\
\text { comentario despectivo. }\end{array}$ & $\begin{array}{l}\text { Tan sólo fue una broma, no } \\
\text { pretendía ofenderla. }\end{array}$ & Ambos se pidieron perdón. \\
\hline Caso 4 & $\begin{array}{l}\text { A excluye a B del grupo de } \\
\text { amigas por "chivarse". }\end{array}$ & $\begin{array}{l}\text { B dice que ella no se ha } \\
\text { "chivado". }\end{array}$ & $\begin{array}{l}\text { Estar juntas en el grupo y no } \\
\text { acusarse. }\end{array}$ \\
\hline Caso 5 & $\begin{array}{l}\text { A inicia una discusión por un } \\
\text { trabajo de clase. }\end{array}$ & B reacciona de modo violento. & $\begin{array}{l}\text { Reflexionar y razonar antes } \\
\text { de actuar violentamente. }\end{array}$ \\
\hline Caso 6 & $\begin{array}{l}\text { A y } B \text { son amigas. A le dice a B } \\
\text { que alguien le ha hecho una foto } \\
\text { comprometida y que está en el } \\
\text { whatsapp pero no le quiere decir } \\
\text { quién le ha hecho la foto. }\end{array}$ & $\begin{array}{l}\text { B se enfada con A y provoca } \\
\text { que la clase esté muy tensa y } \\
\text { acaban con insultos mutuos. }\end{array}$ & $\begin{array}{l}\text { Tratarse respetuosamente } \\
\text { como compañeras de clase. }\end{array}$ \\
\hline Caso 7 & $\begin{array}{l}\text { B continuamente molesta } A \text { en } \\
\text { clase. Los amigos de } B \text { molestan } \\
\text { a A cuando ésta responde. }\end{array}$ & $\begin{array}{l}\text { A continuamente molesta a B. } \\
\text { Los amigos de A también } \\
\text { molestan a B cuando éste } \\
\text { responde. }\end{array}$ & $\begin{array}{l}\text { No harán más comentarios } \\
\text { ofensivos y no se meterán en } \\
\text { las conversaciones del otro. }\end{array}$ \\
\hline
\end{tabular}

En la última mediación, pudieron asistir dos alumnas que habían terminado el proceso de formación y que acudieron como observadoras, paso previo a poder ser ellas mediadoras pro- 
piamente dichas. Tras las mediaciones ninguna pareja volvió a tener conflictos con la persona mediada, aunque algunos de ellos, sí que continuaron teniendo conflictos con otros compañeros que pasaron a solucionarse.

\section{DISCUSIÓN DE LOS RESULTADOS Y CONCLUSIONES}

Como se ha podido comprobar, los programas de mediación como herramienta para la resolución de conflictos y mejora de la convivencia (García-Raga et al., 2016), tiene múltiples beneficios para toda la comunidad educativa en general y para los alumnos mediadores en particular.

En nuestro caso, al estar en el inicio del proceso, no se dispone de datos cuantitativos suficientes para comparar la efectividad de la mediación como mecanismo de mejora de la convivencia. Pero sí se dispone de unas primeras experiencias con la mediación que apuntan hacia los datos que aparecen en la literatura científica (García-Raga y López, 2014). En las mediaciones realizadas por el profesorado las partes del conflicto no han vuelto a tener problemas entre ellos. En algunos casos, han repetido mediación por tener problemas con otro compañero, pero tras la segunda mediación no han vuelto por el servicio de mediación, ya que han aprendido a resolver los problemas relacionales de otro modo.

Conviene recordar que se ha aplicado un programa ya definido para la implantación de la mediación en la institución educativa (Torrego, 2017) pero adaptándolo a las necesidades y a las demandas del centro. Así mismo, el hecho de introducir técnicas y dinámicas de aprendizaje cooperativo (Pujolàs, 2008) ha facilitado considerablemente que el alumnado se conozca y desarrolle competencias y habilidades sociales a favor de la convivencia en el centro educativo.

Respecto a la satisfacción del alumnado que ha realizado la formación en mediación, ha mejorado sus competencias (Silva y Torrego, 2016) de autocontrol, de resolución de conflictos y de mediación tal y como ellos mismos destacan.

En cuanto al desarrollo del programa de mediación entre iguales, al igual que en otros estudios (García-Raga y Grau, 2017), han aparecido dificultades referidas al tiempo que se le dedica a la formación del alumnado, así como a la difusión del servicio de mediación. Ya que sin alumnos bien formados se corre el riesgo de que éstos no sean capaces de conseguir los objetivos que se pretenden al hacer una mediación, es decir, que las dos partes se entiendan, lleguen a un acuerdo y éste se mantenga en el tiempo. Por otra parte, referida a la difusión, si el servicio de mediación no se utiliza por parte del alumnado que presenta algún conflicto, acaba siendo un recurso muy costoso con poco rendimiento que tiende a cansar a las personas implicadas con el riesgo de que se abandone. Por tanto, cuanto más se utilice el recurso mejor, ya que se rentabiliza el coste humano que requiere.

\section{Propuestas de mejora para continuar con la experiencia}

Para que la mediación entre iguales sea una realidad es necesario crear cultura de mediación en todo el centro, ya que si tenemos un servicio de mediación que nadie utiliza es un esfuerzo inútil. Por ello, es necesario difundir la mediación con mayor vehemencia, para que la primera opción del alumnado ante un conflicto sea solicitar el servicio de mediación. Para ello, es inelu- 
dible que toda la comunidad educativa se implique y no sólo el profesorado sensibilizado y que está ejecutando el programa. Por eso, es imprescindible contar con la colaboración del equipo directivo, el claustro de profesores y el AMPA. Por ello, de cara al próximo curso, estos colectivos deberían ser diana para la sensibilización y difusión del programa de mediación, ya que de otra manera, seguirá siendo un programa de un pequeño grupo del claustro sin implicación del resto de la comunidad. El alumnado ha de ser protagonista en el desarrollo de la implantación de la mediación, recogiendo sus demandas y sus sugerencias, animándolos a que sean precursores del cambio cultural del centro.

Además de las mejoras en la difusión, es necesario continuar formando año tras año a nuevo alumnado. Para ello se recogerán las sugerencias y errores detectados en la experiencia llevada a cabo, de tal modo que:

- Las sesiones sean de mayor duración (hora y media como mínimo) y con mayor frecuencia (dos a la semana).

- Realizar más actividades con todos los mediadores para crear un sentido de grupo.

- Hacer más prácticas y de mayor duración.

Se propone:

- Profesor mediador-alumno mediador observa: para consolidar los conocimientos del alumnado mediador, éste participa en las mediaciones reales junto con el profesorado, haciendo de observadores. Por ello, se configura una lista del alumnado que ha asistido a todas las sesiones realizadas hasta el momento, ordenados de mayor edad a menor. El sentido de ordenarlos de este modo es, por un lado, por el grado de madurez asociado a la edad y, por otro lado, al tiempo que permanecerán en el centro. Así que comenzarán los de bachillerato, ya que sólo les queda un año en el centro, mientras que el resto dispone de más años para consolidar su formación. Han de realizar como mínimo dos sesiones reales como observadores antes de pasar a la siguiente sesión.

- Alumno mediador media-profesor observa: el alumnado que ha realizado la sesión anterior se enfrenta a mediaciones reales con el apoyo del profesorado que hace de observadores en esta ocasión. Han de realizar como mínimo dos sesiones con el apoyo del profesorado antes de iniciar las sesiones de manera autónoma. La realización de la mediación autónoma estará supeditada a la evaluación positiva del profesorado observador.

- Introducir técnicas de aprendizaje cooperativo (p.ej. Puzle de Aronson, lectura compartida, Concurso de Vríes, 1-2-4, entre otras) para introducir los aspectos más tediosos y densos del programa referentes a las concepciones teóricas.

- Mayor preparación por parte del profesorado que imparte las clases de formación, ya que en uno de los grupos, ha habido sesiones en la que el profesorado no se ha preparado la sesión previamente y tan sólo ha seguido el guion sobre la marcha creando una sensación de improvisación y de pérdida de tiempo. El profesorado que se comprometa a la formación del alumnado ha de ser consciente de que requiere un esfuerzo y una preparación previa.

- En cuanto a la evaluación de la formación del alumnado, sería conveniente elaborar un formulario donde se recogiese de manera anónima el sentir del alumnado, profesorado y fami- 
lias, referentes a los diversos aspectos que incluye el programa de mediación como por ejemplo la metodología, los materiales, la docencia, etc.

Finalmente, sería importante evaluar el servicio de mediación desde el punto de vista del alumnado atendido, el alumnado mediador, el profesorado y las familias sobre diversos aspectos: la experiencia tenida, el lugar de realización de la mediación, el tiempo dedicado a ello, si ha conseguido resolver el conflicto y la duración de los acuerdos llegados, la difusión del servicio,... Con todos estos datos no sólo se mejorará la calidad de la mediación en el centro, si no que se podrá realizar un estudio en mayor profundidad.

Para conseguir mejorar la convivencia a través de la mediación es necesario mantener una continuidad en el proyecto introduciendo novedades que revivan la ilusión y el entusiasmo. Para ello y como futuras incorporaciones está la “ayuda entre iguales” y la "mediación artística” (Grau y García-Raga, 2018). Respecto a esta última, se podría programar algunos talleres de mediación artística siguiendo las pautas que expone Moreno (2010). Estos talleres irían dirigidos al alumnado menos motivado y más conflictivo. De tal modo que a través de la expresión artística junto con otros compañeros aprendan a expresar y resolver los conflictos de un modo más adecuado al que están acostumbrados. Ambos programas requieren de una formación del profesorado, para ello se podría continuar el próximo curso con un nuevo seminario del CEFIRE.

Siendo conscientes de que la mediación tiene sus limitaciones en la resolución de conflictos interpersonales (Ortega y Del Rey, 1998), son múltiples los Institutos de Secundaria que apuntan por esta medida para mejorar la convivencia en la Comunidad Valenciana desde hace 17 años (García Raga et al., 2017), por lo que son varias las referencias en la aplicación de la mediación dentro de nuestro contexto cultural que avalan apostar por esta clase de proyectos para mejorar la convivencia en el centro.

A modo de conclusión general, este trabajo ha permitido destacar el papel del orientador como coordinador, asesor y formador que puede llegar a desarrollar ante la implantación de un proyecto de mediación. Así como las dificultades, sobre todo en la coordinación de todo el profesorado, teniendo en cuenta que se desarrolla en un IES con más de 650 alumnos y que cuenta con un solo orientador. Por lo tanto, ha permitido desarrollar las habilidades propias del trabajo por programas, en el diseño del programa de mediación, del asesoramiento colaborativo, en las sesiones del seminario y de formador y en la realización de las sesiones del programa de mediación del alumnado. Todo ello con un elevado grado de satisfacción por parte de todos los protagonistas: alumnado y profesorado.

\section{REFERENCIAS}

Aguirre, A. , Benet, A., Moliner, O. , Sales, A. , Sanahuja, A.y Traver, J. A. (2018). Técnicas para la participación democrática. La formación para la convivencia democrática en los procesos de mejora. Servicio de Publicación de la Universitat Jaume I. https://bit.ly/2TKRzLO

Albertín, P. (2007). La formación reflexiva como competencia profesional. Condiciones psicosociales para una práctica reflexiva. El diario de campo como herramienta. Revista de Enseñanza Universitaria, 30, 7-18. https://bit.ly/2X6JxyN 
Alcover, C. (2006). La mediación como estrategia para la resolución de conflictos: Una perspectiva psicosocial. En M. Gonzalo Quiroga (Dir.), Métodos alternativos de solución de conflictos: perspectiva multidisciplinar (pp. 113-129). Dykinson-Servicio de Publicaciones de la URJC.

Aliaga, F.M., Gutiérrez-Braojos, C. y Fernández-Cano, A. (2018). Las revistas de investigación en educación: Análisis DAFO. Revista de Investigación Educativa, 36(2), 563-579. https://bit.ly/2XzPfIF

Boqué, M. (2013). Guía de mediación escolar: programa de comprensivo de actividades de 6 a 16 años. Octaedro.

Corbeña, L. y Romera, C. (s.f.). Cómo poner en marcha paso a paso, un programa de mediación escolar entre compañeros. Departamento de Justicia y Administración Pública del Gobierno Vasco. https:// bit.ly/2TMlUte

Defensor del Pueblo (2007). Violencia escolar: El maltrato entre iguales en la Educación Secundaria Obligatoria 1999-2006. https://bit.ly/3gwGiZi

Delors, J. (coord.) (1996). La educación encierra un tesoro. Informe a la UNESCO de la Comisión Internacional sobre la educación para el siglo XXI. UNESCO/Santillana.

García-Raga, L. , Chiva, I. , Moral, A. y Ramos, G. (2016). Fortalezas y debilidades de la mediación escolar desde la perspectiva del alumnado de educación secundaria. Pedagogía Social. Revista Interuniversitaria, (28), 203-215. https://bit.ly/2TLTIH2

García-Raga, L. y Grau, R. (2017). La mediación en los centros educativos. Percepción desde la coordinación de los equipos de mediación. Universidad de Valencia

García-Raga, L. y López, R. (2014). La convivencia escolar y la construcción de la ciudadanía: balance retrospectivo y desafíos de futuro. Bordón. Revista de Pedagogía, 66(2), 93-106. doi: 10.13042/ Bordon.2014.66206

García-Raga, L., Bo Bonet, R.M. y Boqué Torremorell, M.C. (2017). Percepción del alumnado de Educación Secundaria sobre la mediación escolar en Castellón y Valencia. Revista Complutense de Educación, 28(2), 537-554. https://bit.ly/2ZJjlMp

Grau, R. y García-Raga, L. (2018). ConVivim: aplicación de un programa para aprender a convivir democráticamente. Revista Electrónica de Investigación Educativa, 20(2), 80-92. https://doi. org/10.24320/redie.2018.20.2.1619

Material Para la Mejora de la Convivencia Escolar. Mediación en la resolución de conflictos. Programa de Alumno Ayudante. Junta de Andalucía. Consejería de Educación. https://bit.ly/3gvlnWt

Moreno, A. (2010). La mediación artística: un modelo de educación artística para la intervención social a través del arte. Revista Iberoamericana de Educación, 52(2), 1-9. DOI: 10.35362/rie5221797

Observatorio Estatal de la Convivencia Escolar (2009). Estudio estatal sobre la convivencia escolar en la Educación Secundaria Obligatoria. Avance de resultados. Universidad Complutense de Madrid y el Ministerio de Educación.

Olweus, D. (1998). Conductas de acoso y amenaza entre escolares. Ediciones Morata.

Ortega, R. y Del Rey, R. (1998). Estrategias de intervención con el alumnado en riesgo. En Consejería de Educación y Ciencia de la Junta de Andalucía (Ed.) La convivencia escolar: qué es y cómo abordarla (pp. 197- 220). Consejería de Educación y Ciencia de la Junta de Andalucía.

Peña, B. (2011). Métodos cientificos de observación en educación. Visión Libros. 


\section{E X P E R I E N C I A S}

Pujolàs, P. (2008). Cooperar per aprendre i aprendre a cooperar: el treball en equips cooperatius com a recurs i com a contingut. Suports: Revista Catalana d'educació especial i atenció a la diversitat, 12(1), 21-37. https://bit.ly/3c8PDTH

Pulido, R. , Martín -Seoane, G. y Lucas - Molina, B. (2013). Origines de los programas de mediación escolar: Distintos enfoques que influyen en esta práctica restaurativa. Anales de Psicología, 29(2), 385-392. http://dx.doi.org/10.6018/analesps.29.2.132601

Ruiz Olabuénaga, J. I. (2012). Metodología de la investigación cualitativa. Deusto.

Sánchez, M. (2013). Mediación Educativa Contextualizada. Revista digital de la Asociación CONVIVES, 4, 21-32.

Silva, I. y Torrego, J. C. (2016). El equipo de mediación y el tratamiento de conflictos como herramienta para resolver conflictos. Impacto sobre los alumnos mediadores en un centro de Educación Secundaria. Psicología y Educación: Presente y Futuro. https://bit.ly/2XD7P2r

Torrego, J. C. (2017). Mediación de conflictos en instituciones educativas. Manual para la formación de mediadores. Narcea.

Tuvilla, J. (2004). Cultura de paz: fundamentos y claves educativas. Desclée.

Villanueva L., Usó I. y Adrián J. E. (2013). Los programas de mediación entre iguales: una herramienta eficaz para la convivencia escolar. Apuntes de Psicología, 31, 165-171. https://bit.ly/2TLlROe 